\title{
Should folic acid fortification be mandatory?
}

Nicholas J Wald, director, Wolfson Institute of Preventive Medicine, Barts and The London, Queen Mary's School of Medicine and Dentistry, University of London, London EC1M 6BQ,

n.j.wald@qmul.ac.uk Godfrey P Oakley, professor, Department of Epidemiology, Rollins School of Public Health, Emory University, Atlanta, GA 30322, USA

YES Delay in fortifying flour with folic acid is unjustified. In many countries this public health measure has increased blood folate levels and reduced neural tube defects. When the effect of folic acid on neural tube defects was shown in 1991, prevention was attempted through diet and supplements. ${ }^{1}$ But supplements must be taken before pregnancy is confirmed, and most pregnancies remain unprotected.

Voluntary fortification has proved inadequate. Expert advisory committees considering mandatory fortification with folic acid have concluded that it is necessary, effective, and safe; about 40 countries, including the United States, have adopted this policy.

It is important to reach people who are less well off-they have most to gain from fortification. In Chile, where fortification has achieved a relatively high folic acid intake, neural tube defects were reduced by $43 \%$; in the United States, with a lower fortification level, the reduction was about $20 \%{ }^{2}$ With fortification on a global basis, each year about 250000 children could be saved from spina bifida or anencephaly and the devastating consequences. ${ }^{3}$ This public health opportunity should not be lost.

Evidence indicates other benefits from folic acid fortification: a modest but important protection against cardiovascular disease and a suggested reduction in the evidence of a cleft lip and in the rate of cognitive decline with age. ${ }^{46}$ We consider the scientific validity of four concerns that are raised against fortification.

\section{Link with cancer}

If judgment were to be made, it would be that folic acid prevented cancer, not that it caused it. The US nurses' health study followed 88756 women prospectively, and indicated that long term use of folic acid may substantially reduce the risk of colon cancer. ${ }^{7}$ After 15 years, the relative risk was 0.25 (95\% confidence interval 0.13 to 0.51 ), representing 15 instead of 68 new colon cancers per 10000 women aged 55 to 69 .
A meta-analysis of seven cohort and nine casecontrol studies of colorectal cancer found an overall reduction in risk with folic acid intake. ${ }^{8}$ Smaller cohort studies have been cited to show that low folate may protect against colorectal cancer, ${ }^{9}$ but this interpretation arises from a data subset analysis, is probably due to chance, and is unsupported by the overall results of the trial.

The aspirin-folate prevention trial concluded that folic acid did not result in a significant decrease in large bowel adenomas, ${ }^{10}$ but absence of benefit is not equivalent to the presence of harm. An observed increase of borderline significance was not considered a real effect. Random differences between groups are common in small trials; for example, there were half the number of deaths from all causes in the folic acid group compared with the control group in this trial, which does not mean that folic acid protects against all deaths. A metaanalysis showing a relative risk of $0.99(0.98$ to 1.01) for breast cancer and folate indicates that folic acid neither increases nor decreases the risk of breast cancer. ${ }^{11}$ It is important not to overinterpret marginally significant associations from individual studies (such as one on breast cancer ${ }^{12}$ ); such associations can arise by chance, confounding, or both. The evidence on folic acid and cancer is that there is no harm, and there may be a long term benefit on colorectal cancer.

The case for fortification is sufficiently made on preventing neural tube defects, irrespective of cardiovascular disease prevention. Until recently, the randomised trials of folic acid and cardiovascular disease lacked the statistical power to show that lowering homocysteine by folic acid has a preventive effect, though the HOPE-2 study showed a significant reduction in strokes. ${ }^{13} \mathrm{~A}$ meta-analysis has now confirmed this. ${ }^{14}$ The genetic polymorphism studies also indicate that homocysteine is a cause of cardiovascular disease. ${ }^{4}$

The assertion that folic acid exacerbates B-12 deficiency is without scientific foundation. It is based on reports published more than 50 years ago, when patients with B-12 deficiency had unknowingly been incorrectly treated with folic acid instead of B-12, so the neurological consequences of untreated B-12 deficiency progressed while the macrocytic anaemia (indistinguishable from that due to folate deficiency) improved because high dose folic acid can reverse the arrest of DNA synthesis that causes a B-12 macrocytosis deficiency. The doses of folic acid used in fortification are below those which resolve the anaemia associated with B-12 deficiency. ${ }^{15}$ Moreover, these concerns are unwarranted, because the clinical consequences of B-12 deficiency can be avoided by awareness of the neurological nature of B-12 deficiency, the application of the appropriate biochemical tests, and treatment with B-12.

Synthetic folic acid is ideal for fortification: it is more bio-available than natural folate and, unlike natural folate, is stable in food, even during cooking. It is readily absorbed into the bloodstream-an advantage, as folic acid must pass from the mother's blood to the fetus to be effective. Millions of people have consumed folic acid as supplements for decades before fortification and as a result have had free folic acid in their blood with no credible evidence of any adverse health effects.

\section{Overall assessment}

Folic acid fortification shows clear benefit in preventing spina bifida and anencephaly, with substantial evidence on safety, and no valid indication of harm. Public health authorities have a responsibility to take action, recognising that failure to fortify has serious health consequences; withholding a benefit causes harm. Competing interests: GPO is a co-inventor (while at CDC, compensation, if any, will be under the regulations of $C D C$ ) of a patent that covers adding folic acid to contraceptive pills and has been a paid consultant to Ortho McNeil on the matter of folate. NJW is a co-inventor of a combination pill for the prevention of cardiovascular disease, which optionally may include folic acid. References are in the full version on bmj.com

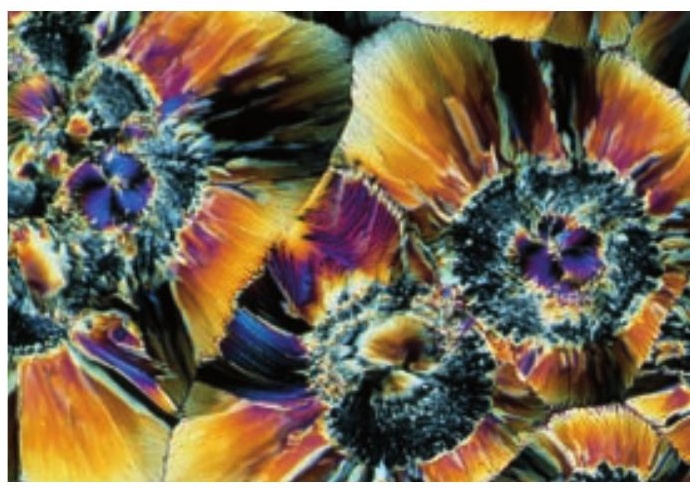


The UK's Food Standards Agency recently recommended mandatory folic acid fortification of some foods. Nicholas Wald and Godfrey Oakley argue that it's a safe effective way of preventing spina bifida and anencephaly - but Richard Hubner and colleagues say that more research is needed

Richard A Hubner, clinical research training fellow, richard.hubner@icr.ac.uk Richard D Houlston, professor of molecular genetics, Institute of Cancer Research, Section of Cancer Genetics, Sutton SM25NG, Kenneth R Muir, professor of epidemiology, University of Nottingham, Division of Epidemiology and Public Health, Queen's Medical Centre, Nottingham NG7 2UH

NO Mandatory fortification with folic acid aims to increase folate intake folate in women during early pregnancy to reduce neural tube defects in their babies. The case for mandatory fortification is strengthened by the purported association of increased folate intake with reduced incidence of cancer. But new data suggest that folate supplements may promote cancer.

Folate metabolism influences several crucial pathways, including DNA synthesis and methylation, abberations of which play a role in carcinogenesis. Altered folate metabolism may disrupt these processes, so folate deficiency and supplementation could influence cancer risk. This may be further complicated by using synthetic folic acid: its effects on folate metabolism are not identical to natural folates. ${ }^{1}$

Epidemiological studies have found that high folate intake is associated with a reduced risk of cancers of the breast, lung, pancreas, oesophagus, stomach, cervix, and the colorectum in particular. ${ }^{2}$ But recent studies have cast doubt over the epidemiological evidence. A large cohort study found an increased risk of colorectal cancer in people with high plasma concentrations of folate, ${ }^{3}$ and a meta-analysis of cohort studies investigating folate intake and breast cancer risk reported no effect. ${ }^{4}$ The inverse relation in some studies of colorectal cancer and folate intake may have been confounded by dietary and lifestyle factors, and adjustment for these may have abolished the association between folate intake and colorectal cancer risk..$^{5}$ The validity of food frequency

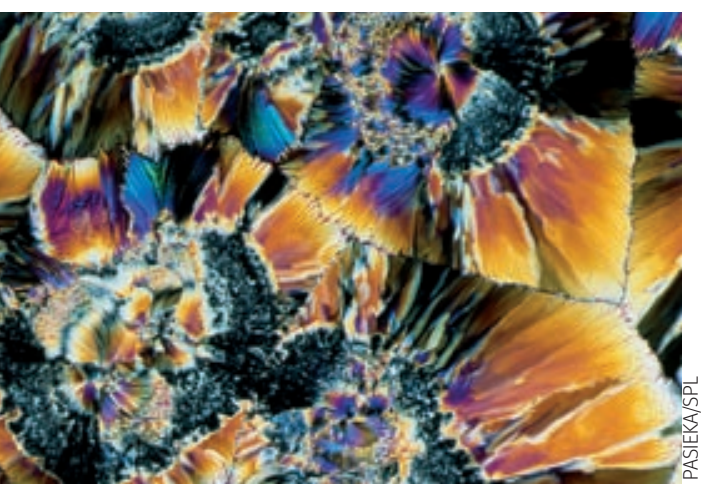

questionnaires assessing folate intake has also been questioned. ${ }^{6}$

Some studies have indictated that folate from foods may have a different effect than folic acid from supplements. A cohort study of 25000 postmenopausal women reported that although folate from food was not associated with risk of breast cancer, high total folate intake, mainly from folic acid supplementation, significantly increased risk by $32 \% .{ }^{7}$ A metaanalysis of cohort studies investigating folate intake and colorectal cancer risk reported a significant reduction in risk in people with high intake of folate from food-but the association was almost null when folate was from diet and folic acid supplements. ${ }^{8}$

\section{Potential cancer promoting effects}

A neoplastic clone of cells has enhanced growth compared to normal tissue. This attribute is exploited by chemotherapeutic drugs, which inhibit folate metabolism enzymes, interrupting DNA synthesis and inhibiting growth of tumours. Extra folate could promote tumour growth by allowing increased DNA synthesis.

Evidence that timing of folic acid supplementation may determine its effects on colorectal carcinogenesis comes from two genetic mouse models of colorectal cancer. ${ }^{910}$ In both models, if intervention was started before lesions developed, moderate folate deficiency enhanced the development of cancer and folic acid supplementation suppressed it-but once a preneoplastic lesion was present, supplementation promoted tumour growth. These studies have led to the hypothesis that in normal epithelial cells folate deficiency promotes neoplastic transformation, which can be avoided by folic acid supplementation, whereas supplementation promotes the growth of existing preneoplastic and neoplastic tissue. Although these studies were in animal models of colorectal cancer, randomised intervention trials in humans support this hypothesis. ${ }^{11-13}$

The aspirin-folate polyp prevention study recruited 1021 people who had colorectal adenoma removed at colonoscopy, randomised to intervention with folic acid ( $1 \mathrm{mg} /$ day) or placebo, and it assessed polyp recurrence by colonoscopy at three and six years. ${ }^{11}$ The mean number of recurrent colorectal adenomas per subject was increased by folic acid supplementation (rate ratio 1.44; 95\% confidence interval 1.03 to 2.02), as was the incidence of advanced colorectal adenoma with high malignant potential $(1.31 ; 0.90$ to 1.89$)$. One explanation for this is that folic acid supplementation promoted the growth of pre-existing aberrant crypt foci or small adenomas and these were missed at initial colonoscopy. If this effect of folic acid is genuine it is a public health concern as more than $25 \%$ of people aged over 50 have asymptomatic colorectal adenomas. ${ }^{14}$

\section{Lowering homocysteine}

Folic acid supplements combined with other $B$ vitamins result in lowered plasma homocysteine, and several randomised intervention studies have investigated their use in preventing cardiovascular disease. A meta-analysis of these studies showed that lowered plasma homocysteine may not prevent ischaemic heart disease (pooled rate ratio $0.96 ; 0.81$ to 1.13 ). ${ }^{15}$ The two largest trials provided data on cancer incidence. Patients randomised to folic acid supplements were $22 \%$ more likely to develop cancer in one trial, ${ }^{12}$ and $36 \%$ and $21 \%$ more likely to develop colorectal cancer and prostate cancer in another. ${ }^{13}$ Although these increases were not statistically significant, cancer incidence was a secondary end point for which both trials were underpowered.

The level of exposure to folic acid from fortification may be crucial, and the low level exposure associated with fortification may not result in any cancer promoting effects. But levels of folate intake after fortification are hard to predict, and the increase in the US since fortification has been twice what was anticipated. ${ }^{16}$

Reducing neural tube defects is a worthy aim, but further investigation of the potential cancer promoting effects of exposure to folic acid in susceptible people is desirable before mandatory fortification starts.

Funding: RAH has a clinical research training fellowship from Cancer Research UK.

\section{Competing interests: None declared.}

References are in the full version on bmj.com Vote now on bmj.com 